\title{
DNMT1 overexpression predicting gastric carcinogenesis, subsequent progression and prognosis: a meta and bioinformatic analysis
}

\author{
Tianmiao Ma ${ }^{1,2}$, Hao Li ${ }^{1}$, Mingjun Sun ${ }^{2}$, Yuan Yuan' ${ }^{1}$ and Li-Ping Sun ${ }^{1}$ \\ ${ }^{1}$ Tumor Etiology and Screening Department of Cancer Institute and General Surgery, The First Affiliated Hospital of China \\ Medical University, Liaoning Provincial Education Department, Key Laboratory of Cancer Etiology and Prevention, China \\ Medical University, Shenyang, Liaoning, China \\ ${ }^{2}$ Digestive Department, The First Affiliated Hospital of China Medical University, Shenyang, Liaoning, China \\ Correspondence to: Li-Ping Sun, email: Ipsun@cmu.edu.cn \\ Yuan Yuan, email: yuanyuan@cmu.edu.cn
}

Keywords: DNMT1, gastric cancer, meta-analysis, bioinformatic analysis

Received: August 02, $2017 \quad$ Accepted: September 20, $2017 \quad$ Published: October 04, 2017

Copyright: Ma et al. This is an open-access article distributed under the terms of the Creative Commons Attribution License 3.0 (CC BY 3.0), which permits unrestricted use, distribution, and reproduction in any medium, provided the original author and source are credited.

\section{ABSTRACT}

DNMT1 is important in maintaining DNA methylation, and participates in the oncogenesis via up- or down-regulation leading to hyper-methylation or hypomethylation. In the meta and bioinformatic analysis, we found that DNMT1 expression was higher in gastric cancer, compared with normal $(p<0.00001)$, para-cancerous $(p=0.0004)$ and dysplasia $(p<0.00001)$ tissues. DNMT1 up-regulation was associated with gender $(O R=2.27, p=0.006)$, differentiation $(O R=0.21, p=0.01)$ and TNM stage (OR $=0.31, p=0.0005)$. Through TCGA database, DNMT1 overexpression increased gastric cancer risk, but unrelated with clinicopathological parameters and prognosis. Kaplan-Meier plotter showed, an increasing expression of DNMT1 was positive for overall survival rates of patients with stage III and IV $(P=0.044 ; P=0.047), N 2$ and N1-3 phases of lymph node metastasis $(P=0.023 ; P=0.032)$, as well as those with or without distant metastasis $(P=0.0052 ; P=0.021)$. For $D N M T 1$ negative patients, the progression-free survival rates was better in patients with Her2+ or Her2- than positive ones $(P=0.00015 ; P=0.031)$. Besides, surgery alone was effective for the overall survival rates in patients with DNMT1 high expression $(P=0.035)$, while 5 -Fu was useful for those with low expression $(P<0.05)$. In conclusion, these findings provided evidence that DNMT1 expression might be employed as a potential marker to indicate gastric carcinogenesis and subsequent progression, even prognosis.

\section{INTRODUCTION}

DNA methylation is the most common epigenetic modification in the mammalian genome [1], involved in several processes during mammalian development including gene expression regulation, genomic imprinting, and $\mathrm{X}$-chromosome inactivation $[2,3]$. Aberrant methylation patterns can be found in human tumors and many abnormalities [4]. DNA methylation is catalyzed by a family of DNA methyltransferases (DNMTs) [5]. It is well known that eukaryotes contain three classes of DNMTs (Dnmt1, Dnmt2, Dnmt3a /Dnmt3b). DNMT1 is a maintenance methylase, which is the most important in the whole process of DNA rmethylation, whereas DNMT3a/DNMT3b is involved in de novo methylation, and DNMT2 is involved in tRNA methylation [6].

DNMT1, the first discovered member of DNMTs [7], which locates in human chromosome 19p13.2, mainly works in maintaining the normal methylation in DNA replication $[8,9]$. In S phase of cell division, DNMT1 is associated with DNA replication sites maintaining the methylation pattern in the newly synthesized strand, which is essential for epigenetic inheritance. And during G2 and M phases, DNMT1 maintains DNA methylation of replication independently to establish the methylation pattern in development $[10,11]$. DNMT1 participates in 
the oncogenesis via up- or down-regulation leading to hyper-methylation or hypo-methylation, respectively [12]. Overexpression of DNMT1 has been detected in several kinds of human cancers. Chen CL [13] reported that DNMT1 mRNA overexpressed in primary and recurrent epithelial ovarian carcinoma. Zhu YM [14] demonstrated that dysregulated expression of DNMT1 in colorectal cancer. Peng DF [15] indicated that increased DNMT1 protein expression was present in multistage pancreatic carcinogenesis from the precancerous stage to malignant progression. And Feng Y [16] reported DNMT1 might play an important role in the early process of lung cancer.

Although some investigators found that the level of DNMT1 expression increased in GC tissues compared with adjacent mucosa or distal normal tissues [17-28], and many reports showed the relationship between DNMT1 expression and different clinicopathological significances in gastric carcinogenesis [17-19, 21, 24, 26-29], but the results were controversial. In addition, the role of DNMT1 in the prognosis of GC was still ambiguous. Therefore, it is necessary to identify eligible studies and perform a meta and bioinformatic analysis to evaluate the carcinogenesis progression and prognosis value of DNMT1 in GC patients.

\section{RESULTS}

\section{Characteristics of eligible studies}

As shown in Table 1, a total of 14 articles on the relationship between DNMT1 expression and risk, clinicopathological parameters as well as prognosis of GC were retrieved for our meta-analysis by immunohistochemistry in PubMed, Web of Science, BIOSIS and CNKI [17-30]. 7 articles contained studies with normal gastric mucosa [17, 18, 20, 21, 24, 26, 27], 2 articles contained studies with gastric precancerous lesions-dysplasia [26, 27] and 7 contained studies with paired adjacent mucosa $[19,20,22-25,28]$. 9 articles showed the differences between DNMT1 expression and clinicopathological characteristics of GC, including age, gender, tumor location, tumor size, differentiation, depth of invasion, TNM staging, Lauren's classification, lymph node metastasis and vascular metastasis $[17,20,21,24$, 26-30]. Additionally, the prognostic significance was analyzed in 3 articles $[17,19,30]$.

\section{Association between DNMT1 expression and GC risk}

In 12 studies with 781 cancers and 890 controls, we analyzed the DNMT1 different expression between GC and non-cancerous mucosa. The results showed, the DNMT1 expression was higher in GC than that in noncancerous mucosa $(p<0.00001$, Figure 1A). And in 7 articles with 454 cancers and 362 normal tissues, we observed the same trend in cancer compared with normal tissues $(P<0.00001$, Figure 1B). When compared with paired adjacent cancerous samples, DNMT1 expression was also observed up-regulation in cancer in 7 studies with 425 cancers and 425 para-cancerous $(p=0.0004$, Figure 1C). In addition, we also found higher DNMT1 expression in GC tissues than that in dysplasia tissues in 2 studies with 132 cancers and 76 dysplasia $(p<0.00001$, Figure 1D).

\section{Association between DNMT1 expression and clinicopathological parameters of GC}

As Figure 2A showed, in GC patients, the higher expression of DNMT1 was in males than that in females $(p=0.006)$, while no significant difference between age groups, no matter the criteria of distinction was 50 or 60 years old ( $p>0.05$, Figure 2B-1, 2B-2). Higher expression of DNMT1 was shown in Stage III-IV than Stage I-II $(p=0.0005$, Figure 2C). In addition, DNMT1 was more expressed in poorly differentiated carcinoma than in well or moderately differentiated carcinoma ( $p=0.01$, Figure 2D). However, DNMT1 expression was not related to tumor location, Laruen's classification, depth of invasion, lymph node metastasis or vascular metastasis of GC ( $p>$ 0.05, Figure 2E-2I).

\section{Heterogeneity analysis}

We did a subgroup analysis based on race. For African, $p=0.90, \mathrm{I}^{2}=0 \%$; for Asian, $p<0.00001, \mathrm{I}^{2}=$ $85 \%$. The results indicated that race might be the main source of heterogeneity.

\section{Publication bias and sensitivity analysis}

Publication bias existed in our meta-analysis (Begg's test, $P=0.040$; Egger's test, $P=0.047$ ). The Funnel Plot graphics showed the publication bias (Figure 3). The sensitivity analysis indicated that before and after the deletion of each study, the results were stable, demonstrating the stability of the meta-analysis (Figure 4).

\section{Association between DNMT1 expression and the risk, clinicopathological and prognostic significance of GC using informatics analysis}

First, we integrated the raw data from TCGA database to investigate the association between DNMT1 expression and the risk of GC. The results showed DNMT1 expression was higher in GC than that in normal tissues $(P<0.0001)$. However, the abnormal expression of DNMT1 had no significant relations with some clinicopathological parameters, such as gender, age, TNM stage, histological type and prognosis (Table 2). 
Table 1: Basic characteristics of eligible studies

\begin{tabular}{|c|c|c|c|c|c|c|c|c|c|c|}
\hline Study & Year & Country & Ethnicity & $\begin{array}{l}\text { Antibody } \\
\text { company }\end{array}$ & $\begin{array}{c}\text { No. of Cases } \\
(\mathbf{M}+/ \mathbf{M}-)\end{array}$ & $\begin{array}{c}\text { No. of } \\
\text { Control } \\
(\mathbf{M}+/ \mathbf{M}-)\end{array}$ & $\begin{array}{l}\text { Risk to } \\
\text { cancer }\end{array}$ & $\begin{array}{l}\text { Risk to } \\
\text { aggress }\end{array}$ & Outcome & Quality \\
\hline Gao Y [22] & 2016 & China & Asian & - & $90 / 30$ & $7 / 113$ & Up & & & 6 \\
\hline F.Ksiaa [17] & 2015 & Tunisia & African & DakoCytomation & $24 / 23$ & $0 / 47$ & $\mathrm{Up}$ & $\mathrm{Neg}$ & Neg & 7 \\
\hline Liang YY [24] & 2015 & China & Asian & BIOSS & $43 / 17$ & $59 / 61$ & Up & Neg & & 8 \\
\hline F.Ksiaa [18] & 2014 & Tunisia & African & DakoCytomation & $20 / 23$ & $0 / 43$ & Up & & & 6 \\
\hline Cao XY [19] & 2014 & China & Asian & Santa Cruz & $51 / 34$ & $32 / 53$ & Up & Neg & Neg & 7 \\
\hline Lu YO [23] & 2013 & China & Asian & - & $15 / 5$ & $6 / 14$ & Up & & & 7 \\
\hline Jiang XJ [25] & 2012 & China & Asian & - & $56 / 12$ & $10 / 58$ & Up & & & 8 \\
\hline Mutze K [30] & 2011 & Germany & European & Santa Cruz & $105 / 22$ & $0 / 0$ & Up & Pos & Pos & 6 \\
\hline Sun N [26] & 2011 & China & Asian & BIOSS & $48 / 14$ & $36 / 47$ & $\mathrm{Up}$ & Neg & & 8 \\
\hline Han J [29] & 2010 & China & Asian & Abcam & $55 / 40$ & $0 / 0$ & Up & & & 6 \\
\hline Liu B [27] & 2009 & China & Asian & ALEXIS & $49 / 21$ & $14 / 46$ & $\mathrm{Up}$ & Pos & & 7 \\
\hline Ding WJ [20] & 2008 & China & Asian & Santa Cruz & $31 / 7$ & $19 / 57$ & $\mathrm{Up}$ & Neg & & 7 \\
\hline Liu T [28] & 2007 & China & Asian & Santa Cruz & $32 / 2$ & $29 / 5$ & Up & & & 7 \\
\hline Etoh T [21] & 2004 & Japan & Asian & Santa Cruz & $97 / 37$ & $0 / 134$ & Up & Pos & & 6 \\
\hline
\end{tabular}

Table 2: Association between DNMT1 expression and the pathologic parameters and prognosis of GC from TCGA database

\begin{tabular}{lcc}
\hline & $\boldsymbol{N}$ & $\boldsymbol{P}$ \\
\hline Gender & & 0.412 \\
male & 243 & \\
female & 137 & \\
Age & & 0.856 \\
$\quad>60$ & 255 & \\
$\leq 60$ & 125 & 0.368 \\
TNM stage & & \\
I-II & 167 & \\
III-IV & 190 & 0.424 \\
histological type & & \\
intestinal & 168 & \\
diffuse & 63 & 0.194 \\
prognosis & & \\
dead & 150 & \\
alive & 230 & \\
\hline
\end{tabular}

Second, using the Kaplan-Meier plotter, we found that DNMT1 mRNA expression was not associated with the overall survival (OS) or progression-free survival (PFS) in all GC patients $(P>0.05$, Figure 5A, 5B). However, DNMT1 overexpression in patients with lymph node metastasis (N2, N1-3) and distant metastasis (M0, M1) showed a long overall survival time $(P<0.05$, Figure $5 \mathrm{C}-$ $5 \mathrm{~F})$, and those in stage III and stage IV also showed a good outcome for OS $(P<0.05$, Figure $5 \mathrm{G}, 5 \mathrm{H})$. For DNMT1 negative patients, the PFS with Her2+ or Her2- was better than those in positive ones $(P<0.05$, Figure 5I, 5J).
In addition, surgery alone was effective for the OS of patients with DNMT1 up-regulation $(P=0.035$, Figure $5 \mathrm{~K})$, but 5 -Fu were useful for the patients with DNMT1 negative expression $(P<0.05$, Figure $5 \mathrm{~L}, 5 \mathrm{M})$. Therefore, DNMT1 overexpression might be considered as a reference marker to select clinical therapeutic regimen.

\section{DISCUSSION}

DNMT1, as the most important enzyme of maintaining DNA methylation, widely exists in 
many kinds of tumors. However, the relationship between DNMT1 overexpression and the risk, clinicopathological parameters and prognostic of GC is indefinite. In the present study, we used a meta-analysis to summarize literature review on the correlation between DNMT1 expression and GC risk, as well as its clinical pathological parameters. On account of a limited data with the GC prognosis, we made a bioinformatic analysis of TCGA database to explore the association of DNMT1 overexpression with clinical outcomes of GC patients.

As the results showed in our meta-analysis, DNMT1 expression in GC was much higher than that in noncancerous, which suggested that DNMT1 could be a good biomarker for diagnosis of GC. Furthermore, we compared the difference of DNMT1 expression between normal, para-

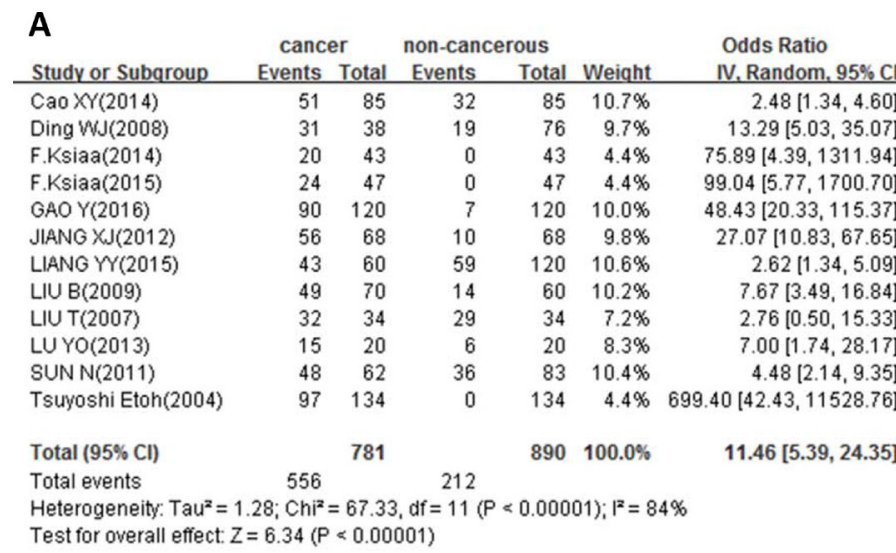

\begin{tabular}{|c|c|c|c|c|c|c|}
\hline Study or Subqroup & $\begin{array}{c}\text { cance } \\
\text { Events }\end{array}$ & Total & $\begin{array}{l}\text { norma } \\
\text { Events }\end{array}$ & $\begin{array}{l}\text { al } \\
\text { Total }\end{array}$ & Weight & $\begin{array}{l}\text { Odds Ratio } \\
\text { IV. Random, } 95 \% \mathrm{Cl}\end{array}$ \\
\hline Ding WJ(2008) & 31 & 38 & 4 & 38 & $18.0 \%$ & $37.64[10.04,141.11]$ \\
\hline F.Ksiaa(2014) & 20 & 43 & 0 & 43 & $10.9 \%$ & $75.89[4.39,1311.94]$ \\
\hline F.Ksiaa(2015) & 24 & 47 & 0 & 47 & $10.9 \%$ & $99.04[5.77,1700.70]$ \\
\hline LIANG YY(2015) & 43 & 60 & 22 & 60 & $20.5 \%$ & $4.37[2.03,9.42]$ \\
\hline LIU B(2009) & 49 & 70 & 0 & 20 & $10.9 \%$ & $94.40[5.46,1633.06]$ \\
\hline SUN N(2011) & 48 & 62 & 3 & 20 & $17.8 \%$ & $19.43[4.97,76.01]$ \\
\hline Tsuyoshi Etoh(2004) & 97 & 134 & 0 & 134 & $11.0 \%$ & $699.40[42.43,11528.76]$ \\
\hline Total $(95 \% \mathrm{Cl})$ & & 454 & & 362 & $100.0 \%$ & $39.35[10.53,147.09]$ \\
\hline Total events & 312 & & 29 & & & \\
\hline \multicolumn{7}{|c|}{ Heterogeneity: $\mathrm{Tau}^{2}=2.05 ; \mathrm{Chi}^{2}=23.56, \mathrm{df}=6(\mathrm{P}=0.0006) ; I^{2}=75 \%$} \\
\hline
\end{tabular}
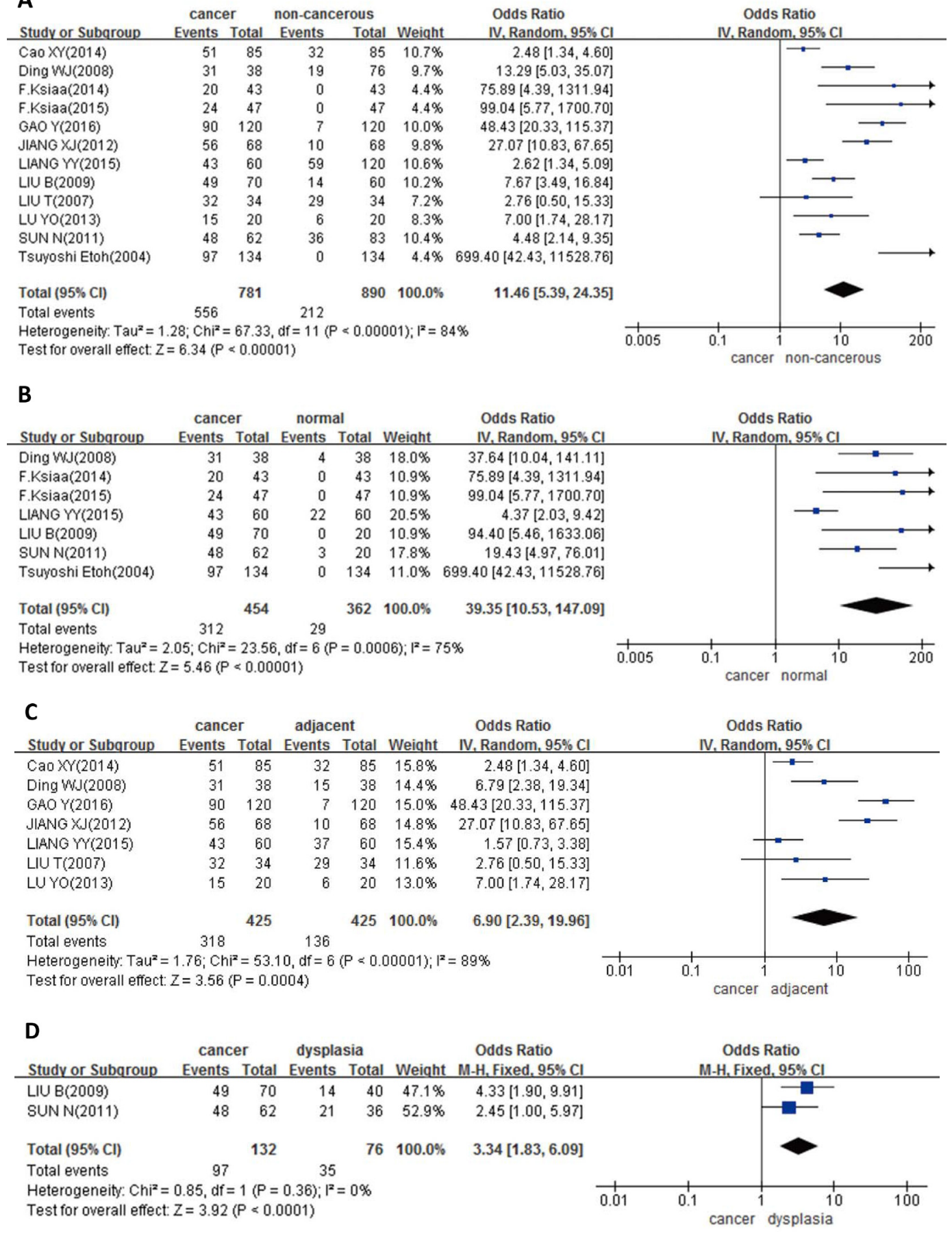

Figure 1: Forest plot for the relationship between DNMT1 expression and GC risk in different subgroups. (A) Gastric carcinogenesis (cancer vs. non-cancerous); (B) gastric carcinogenesis (cancer vs. normal); (C) gastric carcinogenesis (cancer vs. adjacent); (D) gastric carcinogenesis (cancer vs. dysplasia). 


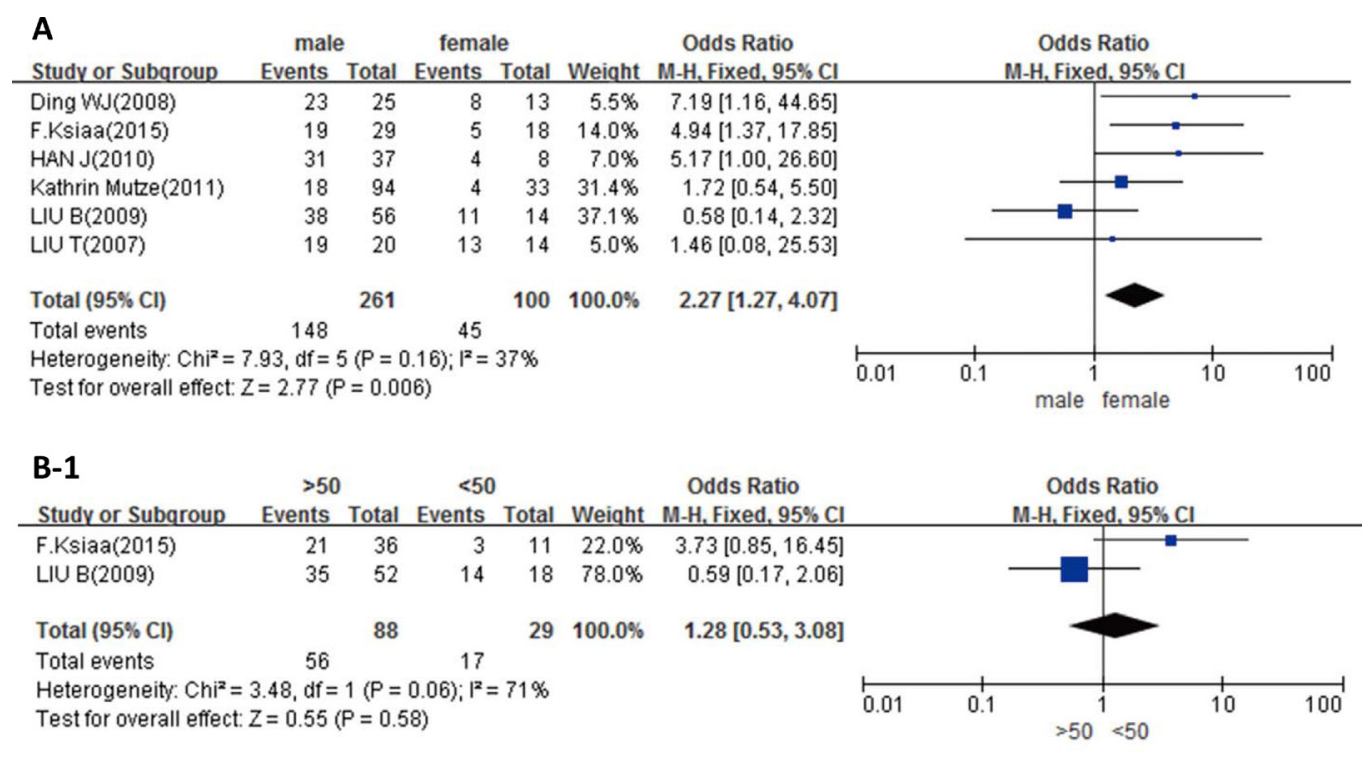

\section{B-2}

\begin{tabular}{|c|c|c|c|c|c|c|c|c|c|c|c|}
\hline & $>60$ & & $<60$ & & & Odds Ratio & & & Odds Ratio & & \\
\hline Study or Subqroup & Events & Total & Events & Total & Weiqht & M-H, Fixed, 95\% Cl & & & M-H, Fixed, 95\% Cl & & \\
\hline Ding WJ(2008) & 19 & 23 & 12 & 15 & $11.4 \%$ & $1.19[0.23,6.26]$ & & & & & \\
\hline HAN J(2010) & 7 & 10 & 28 & 35 & $16.9 \%$ & $0.58[0.12,2.85]$ & & & & & \\
\hline Kathrin Mutze(2011) & 60 & 72 & 45 & 55 & $38.4 \%$ & $1.11[0.44,2.80]$ & & & & & \\
\hline LIANG YY(2015) & 32 & 39 & 15 & 21 & $15.8 \%$ & $1.83[0.52,6.39]$ & & & & & \\
\hline SUN N(2011) & 33 & 41 & 15 & 21 & $17.5 \%$ & $1.65[0.49,5.60]$ & & & & & \\
\hline Total $(95 \% \mathrm{Cl})$ & & 185 & & 147 & $100.0 \%$ & $1.24[0.71,2.16]$ & & & & & \\
\hline Total events & 151 & & 115 & & & & & & & & \\
\hline $\begin{array}{l}\text { Heterogeneity: } \mathrm{Chi}^{2}= \\
\text { Test for overall effect: }\end{array}$ & $\begin{array}{l}.51, \mathrm{df}= \\
=0.75(\end{array}$ & $\begin{array}{l}4(\mathrm{P}=0 \\
=0.4\end{array}$ & 83); $F^{2}=$ & & & & 0.01 & 0.1 & $>60^{1}<60$ & 10 & 100 \\
\hline
\end{tabular}

\section{C}

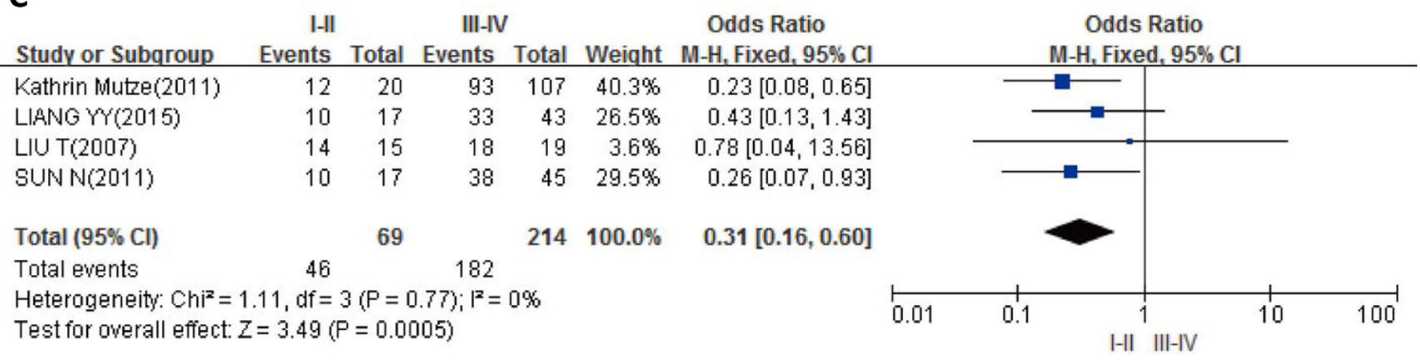

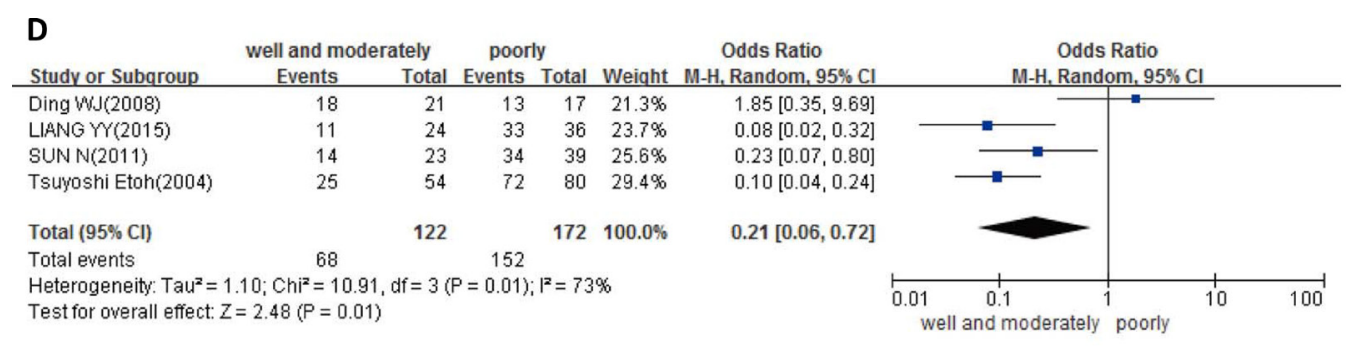

\section{E}

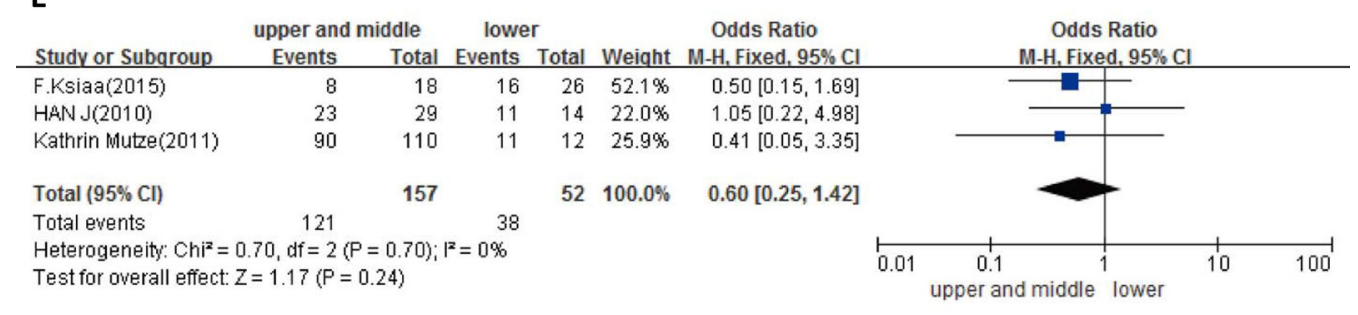



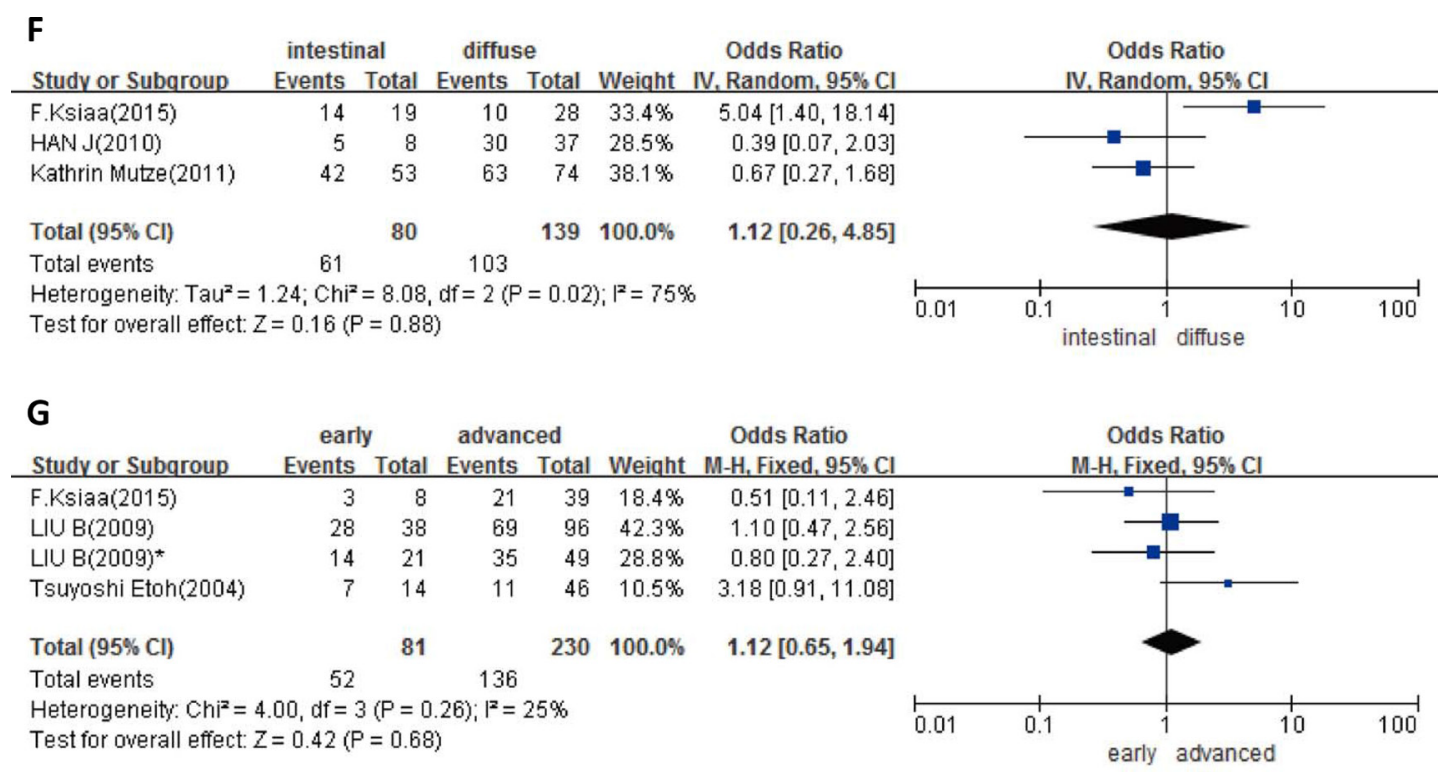

\begin{tabular}{|c|c|c|c|c|c|c|c|c|c|c|c|}
\hline $\begin{array}{l}\text { H } \\
\text { Studv or Subqroup }\end{array}$ & $\begin{array}{c}\text { LN+ } \\
\text { Events }\end{array}$ & Total & $\begin{array}{l}\mathrm{LN} \text { - } \\
\text { Events }\end{array}$ & Total & Weight & $\begin{array}{c}\text { Odds Ratio } \\
\text { M- } \mathrm{H} \text {, Fixed, } 95 \% \mathrm{Cl}\end{array}$ & & & $\begin{array}{l}\text { Odds Ratio } \\
\text { M-H, Fixed, } 95 \% \mathrm{Cl}\end{array}$ & & \\
\hline Ding WJ(2008) & 16 & 20 & 15 & 18 & $9.1 \%$ & $0.80[0.15,4.18]$ & & & & & \\
\hline F.Ksiaa(2015) & 9 & 21 & 15 & 26 & $22.1 \%$ & $0.55[0.17,1.76]$ & & & & & \\
\hline LIANG YY(2015) & 38 & 47 & 9 & 13 & $7.8 \%$ & $1.88[0.47,7.49]$ & & & & & \\
\hline LIU B(2009) & 33 & 41 & 16 & 29 & $10.5 \%$ & $3.35[1.16,9.71]$ & & & & & \\
\hline LIU T(2007) & 21 & 21 & 11 & 13 & $0.9 \%$ & $9.35[0.41,211.62]$ & & & & & \\
\hline SUN N(2011) & 39 & 49 & 9 & 13 & $8.4 \%$ & $1.73[0.44,6.80]$ & & & & & \\
\hline Tsuyoshi Etoh(2004) & 37 & 53 & 60 & 81 & $41.3 \%$ & $0.81[0.38,1.75]$ & & & & & \\
\hline Total $(95 \% \mathrm{Cl})$ & & 252 & & 193 & $100.0 \%$ & $1.26[0.81,1.96]$ & & & & & \\
\hline Total events & 193 & & 135 & & & & & & & & \\
\hline \multicolumn{7}{|c|}{$\begin{array}{l}\text { Heterogeneity: } \text { Chi }^{2}=8.87, d f=6(P=0.18) ; I^{2}=32 \% \\
\text { Test for overall effect: } Z=1.01(P=0.31)\end{array}$} & 0.01 & 0.1 & $\mathrm{LN}^{1}{ }^{1} \mathrm{LN}-$ & 10 & $100^{\circ}$ \\
\hline
\end{tabular}

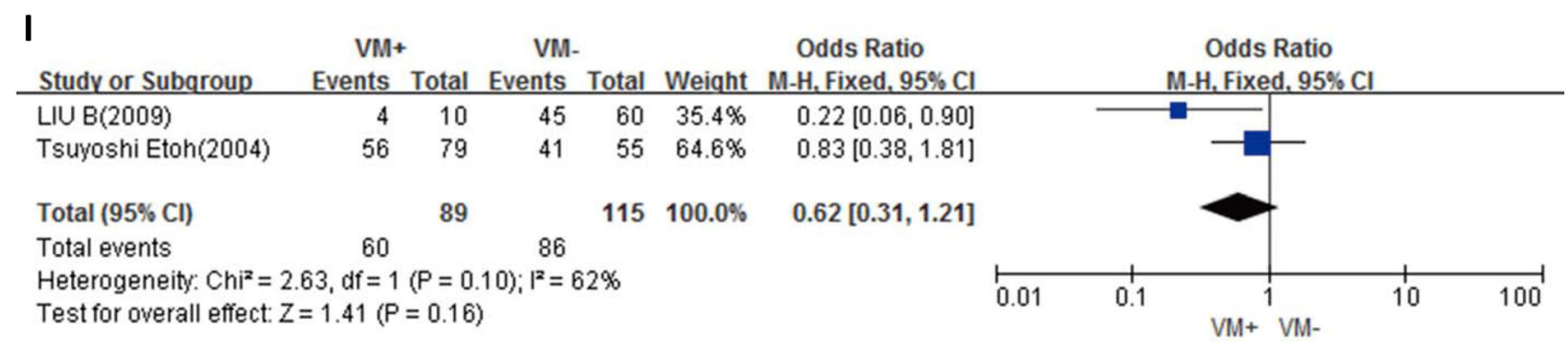

Figure 2: Forest plot for the relationship between DNMT1 expression and clinicopatholoiocal parameters of GC. (A) Correlation between sex and DNMT1 expression (male vs. female); (B-1) correlation between age and DNMT1 expression ( $>50$ vs. $<50)$; (B-2) correlation between age and DNMT1 expression (> 60 vs. $<60$ ); (C) correlation between TNM staging and DNMT1 expression (I-II vs. III-IV); (D) correlation between differentiation and DNMT1 expression (well and moderately vs. poorly); (E) correlation between tumor location and DNMT1 expression (upper and middle vs. lower); (F) correlation between Lauren's classification and DNMT1 expression (intestinal type vs. diffuse type); (G) correlation between depth of tumor and DNMT1 expression (early vs. advanced); (H) correlation between lymph node metastasis (LN) and DNMT1 expression (LN+ vs. LN-); (I) correlation between vascular metastasis (VM) and DNMT1 expression (VM+ vs. VM-).

cancerous, dysplasia tissues and GC tissues, respectively. We could speculate from the results above that with the increase of the severity of the gastric disease, DNMT1 protein expression increased at the same time. The results were basically consistent with the literatures we included.

We also found that DNMT1 overexpression was related to gender, differentiation and TNM stage in GC.
DNMT1 preferentially expressed in men than women according to seven articles of our meta-analysis, and two of them got the same results $[17,20]$. It's probably because that the mean age of the patients in these two reports were older than others. The expression of DNMT1 in patients with poor differentiation was higher than well and moderately differentiation, which was consistent with 
Liang [24], Sun [26] and Etoh [21]. Moreover, the upregulated expression of DNMT1 was very common in IIIIV stage when compared to that in I-II stage, although only one article revealed such difference [26]. However, as we can see, this overexpression of DNMT1 was not associated with age, tumor location, Lauren's classification, depth of invasion, lymph lode metastasis and vascular metastasis. Majority researches were similar to the results above,

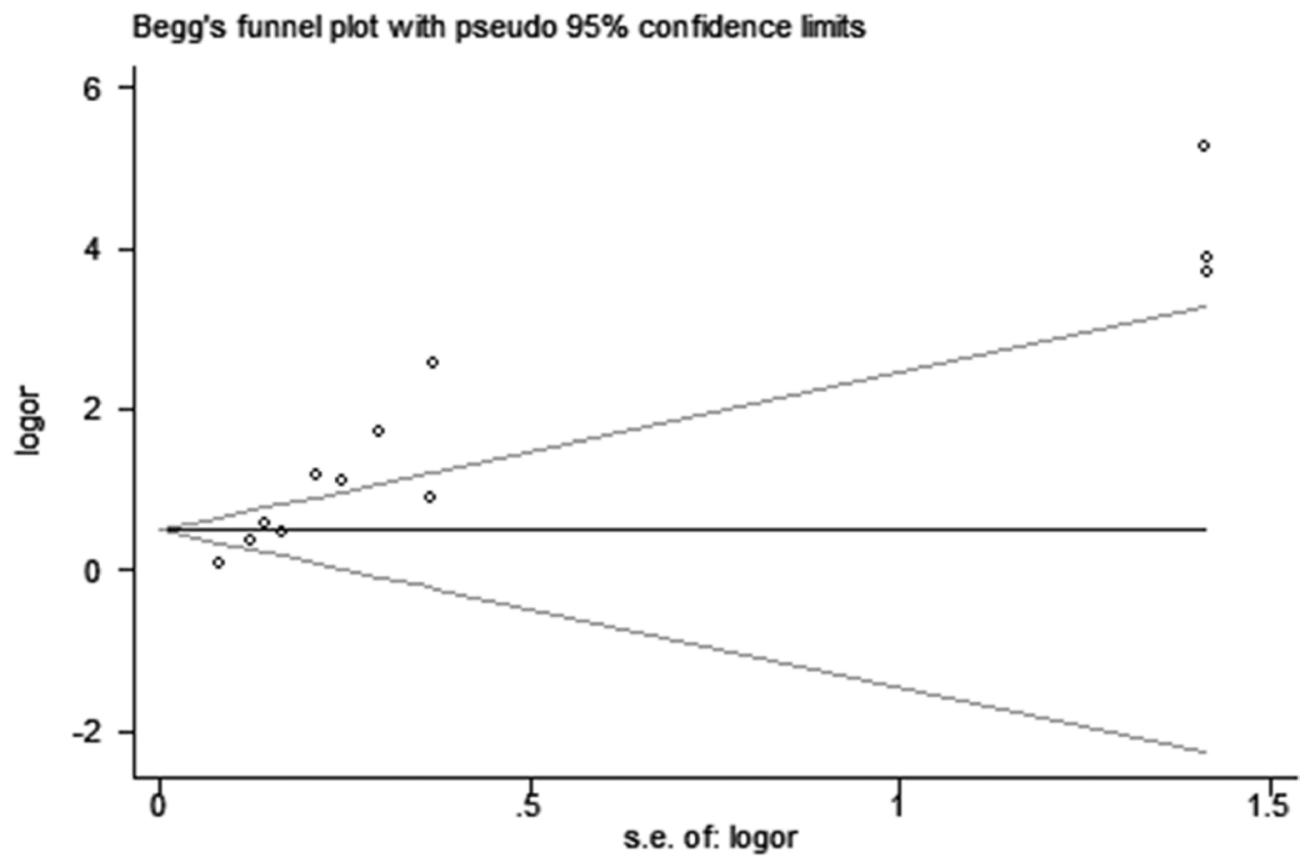

Figure 3: The funnel plot graphics of publication bias. The bias was analyzed about risk degrees of DNMT1 expression in gastric mucosa for gastric carcinogenesis.

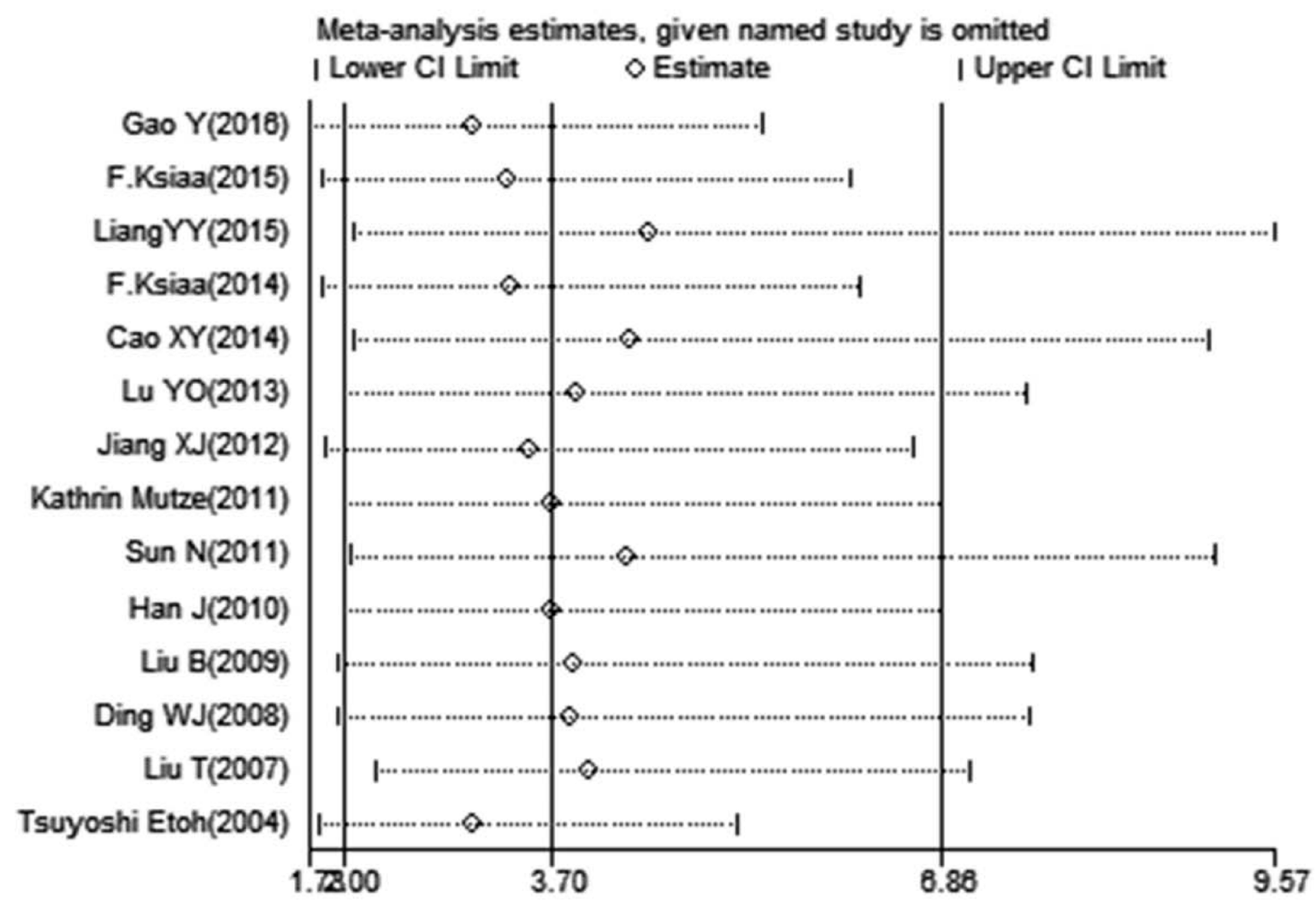

Figure 4: Sensitivity analysis. 

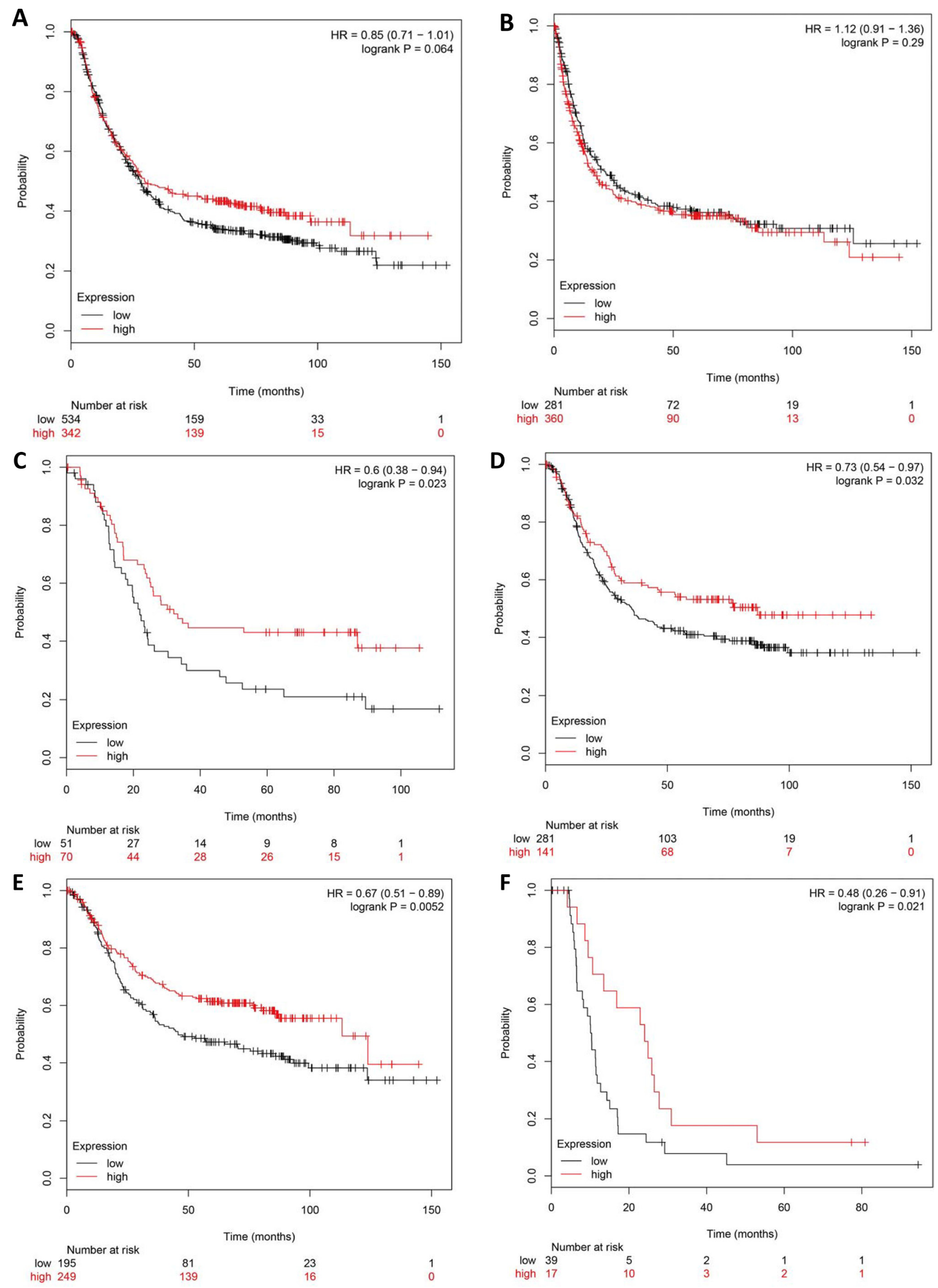

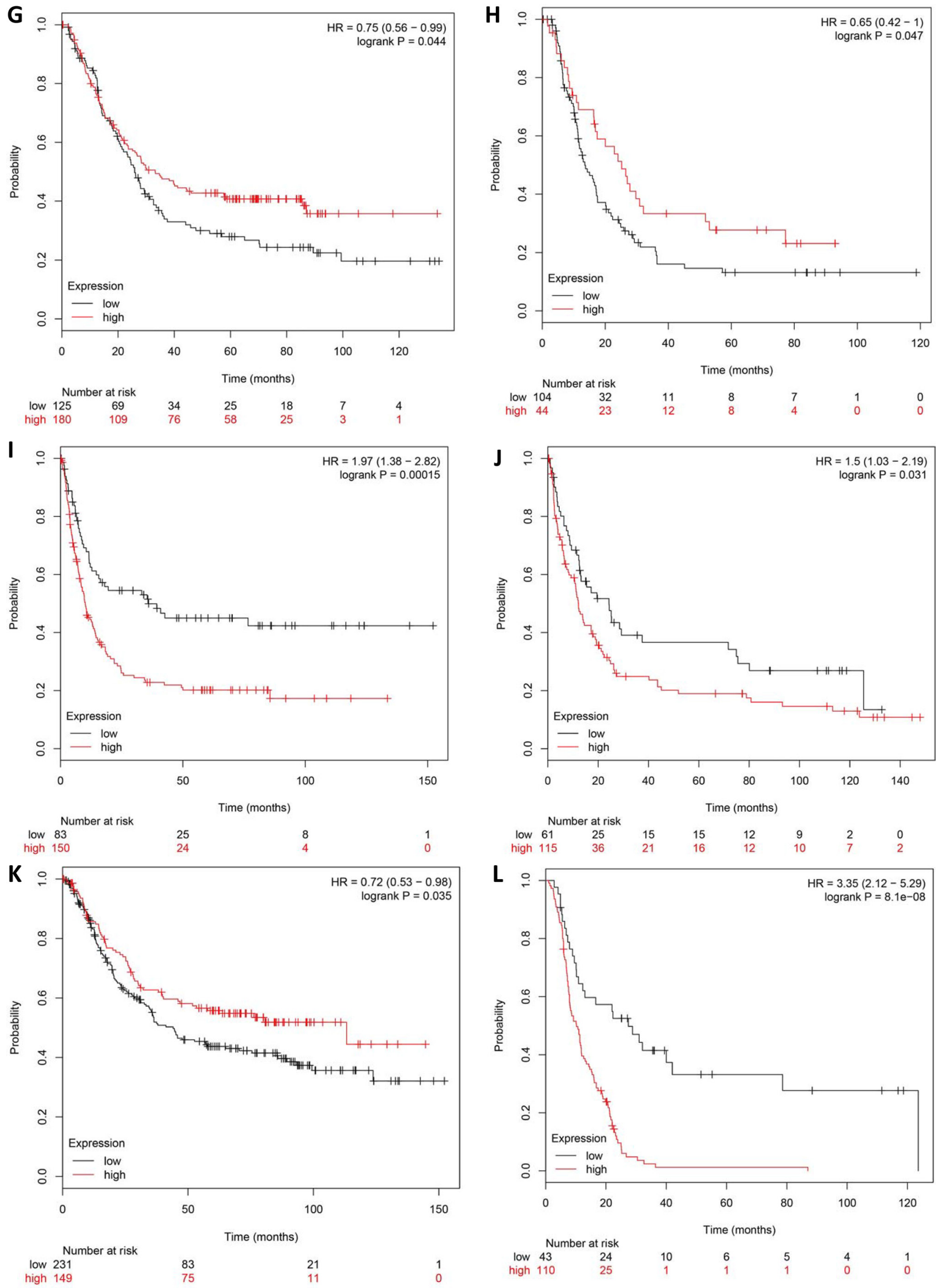


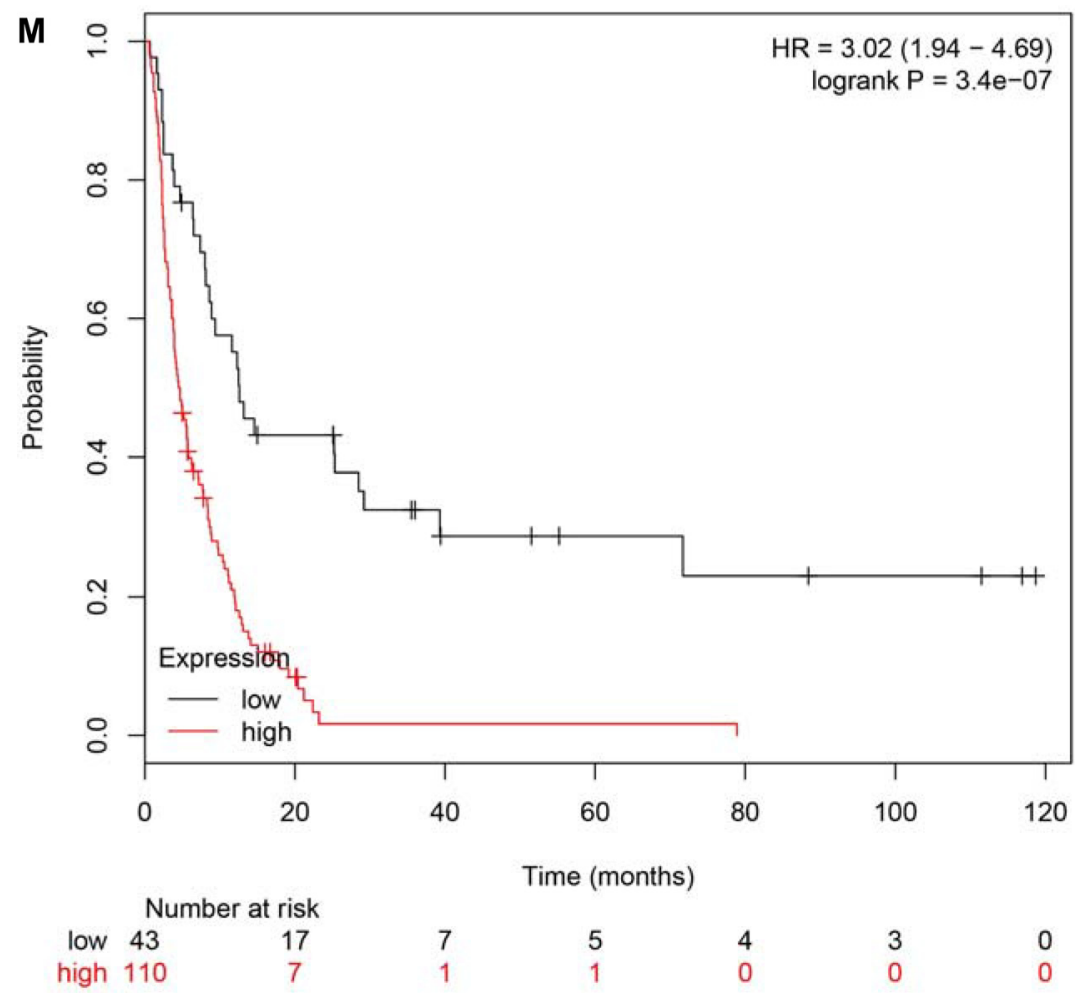

Figure 5: The prognostic significance of DNMT1 mRNA in the patients with gastric cancer according to the database from Kaplan Meier plotter. DNMT1 mRNA expression was not related to both overall (A) and progression-free (B) survival rates of the patients with gastric cancer. DNMT1 mRNA expression has relation with OS in N2 (C), N1-3 (D), M0 (E), M1 (F), Stage 3 (G), Stage 4 (H), Surgery alone $(\mathbf{K}), 5-\mathrm{Fu}(\mathbf{L}), 5-\mathrm{Fu}(\mathbf{M})$, and with PFS in Her2+ (I) and Her2- (J).

whereas some studies verified that patients with lymph node metastasis may increase DNMT1 expression.

Furtherly, we used bioinformatic databases to investigate the role of DNMT1 expression in the progression of GC. Using TCGA database, we found that the expression of DNMT1 was highly correlated with the risk of $\mathrm{GC}$, as the statistically significant for the difference of DNMT1 expression between the cancer group and the normal group. We also analyzed the relationship between the expression of DNMT1 and parts of pathological parameters of GC through TCGA, although the results were not completely the same as our meta-analysis, there were still some similarities. On the basis of TCGA results, no differential expression of DNMT1 has been found between age (take 60 years as the line) and histological type, which was in accordance with our consequence. As for the difference between TCGA and our meta results in gender and TNM stage, this might because the patients were mainly from hospital, and most of them were Chinese.

Analysis on databases of Kaplan-Meier plotter showed that DNMT1 expression has no relation with prognosis. However, we found a relationship between DNMT1 expression and prognostic significance with clinical parameters, such as lymph node metastasis, distant metastasis and TNM stage. Besides, surgery alone had effectivity for patients with DNMT1 positive expression, but $5-\mathrm{Fu}$ or other adjuvant was helpful for those with DNMT1 negative expression. Mutze K [30] also reported that GC patients with low DNMT1 expression would have a good outcome after 5-Fu based neoadjuvant chemotherapy. These results indicated that DNMT1 can serve as a biomarker for clinical therapeutic regimen.

There are several limitations should be noted in the study. First, patient populations were limited, as patients mainly from Asian, especially Chinese. Additionally, the patients basically came from the hospital, which also caused the patients bias. Second, the potential publication bias stems from published results being predominantly positive. Third, we mainly included the studies that the method of immunohistochemistry with full-texts, however there were still some unpublished personal data or meeting abstracts unavailable. Fourth, this limited sample size may affect the power to detect the associations in some articles.

In conclusion, we demonstrated the DNMT1 abnormal overexpression might be employed as a good potential marker for prediction of $\mathrm{GC}$, and related with clinicopathological parameters, as well as the prognosis of GC. Further large-scale and well-designed studies are still needed to confirm the results of our meta and bioinformatic analysis. 


\section{MATERIALS AND METHODS}

\section{Identification of eligible studies and selection criteria}

We performed a publication search using PubMed, Web of Science, BIOSIS, and CNKI updated on Jun 5th, 2017, and only the human studies were searched. The following search terms were used: (DNMT1 OR MCMT) AND (gastric OR stomach) AND (cancer OR carcinoma OR adenocarcinoma)). Searching was done without restriction on language or publication years.

Inclusion criteria for studies: (1) relevant articles to observe the DNMT1 protein expression in GC (2) papers to compare the DNMT1 expression with risk and clinicopathologic significance of $\mathrm{GC}$ by immunohistochemistry. Exclusion criteria included: (1) abstract, comment, review and meeting; (2) duplication studies of previous reports; (3) Western blot, RT-PCR, or other detection method for DNMT1 expression; (4) lack of sufficient information. Finally, 15 articles were selected for our meta-analysis (Figure 6).

\section{Data extraction}

Based on the inclusion criteria, two independent reviewers (TM Ma and LP Sun) extracted information from all eligible publications. We summarized the information and statistical data that extracted from full- text format. The following information was extracted in each study: name of first author, year of publication, country, ethnicity, antibody company, numbers of cases and controls, risk to cancer, aggress and outcome. Any disagreement was solved through discussion until the two reviewers reached a consensus (Table 1).

\section{Quality score assessment}

Two reviewers (TM Ma and LP Sun) independently assessed the quality of the 15 studies by using Newcastle Ottawa Scale (NOS). The scale contains three parts: sample selection, comparability and exposure outcome. Based on the quality score criteria, the higher scores indicated, the better quality 10 points is our top score, and more than 5 points was accepted in our meta-analysis.

\section{Bioinformatic analysis}

We downloaded 443 patients' data from stomach database in Cancer Genome Atlas (TCGA) by TCGAassembler with $\mathrm{R}$ software. Among them, 408 patients were with the normal stomach or gastric cancer, and 380 patients had clinicopathologic significance and prognostic information. We just picked out 408 cases to evaluate the GC risk, and 380 cases to analyze the clinicopathologic significance and prognostic of GC. Furthermore, the prognostic significance of DNMT1 mRNA was analyzed using Kaplan-Meier plotter.

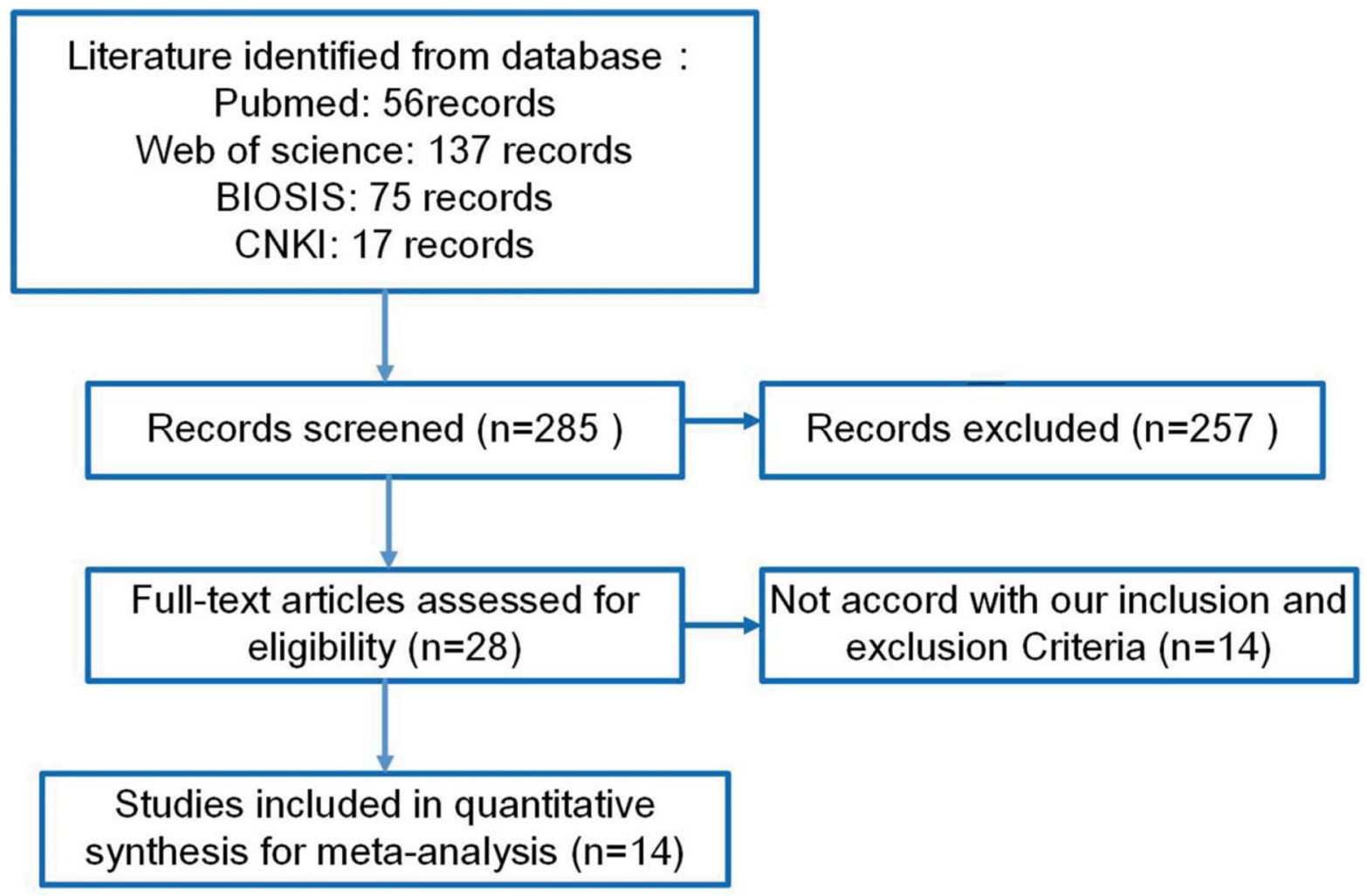

Figure 6: Flow chart of the selection process in this meta-analysis. 


\section{Statistics analysis}

Statistical analyses were carried out by Review Manager vision 5.3 (The Cochrane Collaboration, Nordic Cochrane Centre Copenhagen, Denmark).The relationship between DNMT1 expression and cancer risk, different clinicopathologic characteristics were assessed by odds ratios (OR) with $95 \%$ confidence intervals (CI). Statistical significance of the pooled OR was determined by $Z$ test, and it was meaningful when $P$-value $<0.05$. Heterogeneity effect was quantified by the Chisquarebased $Q$ test and the $\mathrm{I}^{2}$ test. An $\mathrm{I}^{2}<50 \%$ or $P$-value $>0.10$, indicated no significant heterogeneity, and a fixed-effects model (Mantel-Haenszel method) was used. Otherwise, the random-effects model (DerSimonian and Laird method) was used. We explored the possible source of statistical heterogeneity by subgroup analysis according to the ethnicity of patients, and sensitivity analysis. Publication bias and sensitivity analysis were calculated by State vision 11.0 (State corporation, College Station, TX, USA). Publication bias was estimated by Begg's test and Egger's test to assess funnel plot asymmetry. Data from TCGA database was dealt by $t$-test using SPSS vision 22.0 (IBM SPSS statistics, Armonk, NY, USA), and $P$-value $<0.05$ was considered as statistically significant.

\section{CONFLICTS OF INTEREST}

None of the authors has a commercial or other association that might pose a conflict of interest.

\section{FUNDING}

This work was supported by grants from the National Key R\&D Program (ref no. 2016YFC1303202); and the Key Laboratory of Cancer Intervention in Liaoning Province (ref no. LS201605).

\section{REFERENCES}

1. Jones PA, Takai D. The role of DNA methylation in mammalian epigenetics. Science. 2001; 293:1068-1070.

2. Lister R, Pelizzola M, Dowen RH, Hawkins RD, Hon G, Tonti-Filippini J, Nery JR, Lee L, Ye Z, Ngo QM, Edsall L, Antosiewicz-Bourget J, Stewart R, et al. Human DNA methylomes at base resolution show widespread epigenomic differences. Nature. 2009; 462:315-322.

3. Bird A. DNA methylation patterns and epigenetic memory. Genes \& development. 2002; 16:6-21.

4. Koh KP, Rao A. DNA methylation and methylcytosine oxidation in cell fate decisions. Current opinion in cell biology. 2013; 25:152-161.

5. Moore LD, Le T, Fan G. DNA methylation and its basic function. Neuropsychopharmacology. 2013; 38:23-38.
6. Dan J, Chen T. Genetic Studies on Mammalian DNA Methyltransferases. Advances in experimental medicine and biology. 2016; 945:123-150.

7. Wang Y, Jorda M, Jones PL, Maleszka R, Ling X, Robertson HM, Mizzen CA, Peinado MA, Robinson GE. Functional CpG methylation system in a social insect. Science. 2006; 314:645-647.

8. Klein CJ, Bird T, Ertekin-Taner N, Lincoln S, Hjorth R, Wu Y, Kwok J, Mer G, Dyck PJ, Nicholson GA. DNMT1 mutation hot spot causes varied phenotypes of HSAN1 with dementia and hearing loss. Neurology. 2013; 80:824-828.

9. Kanwal R, Gupta S. Epigenetic modifications in cancer. Clinical genetics. 2012; 81:303-311.

10. Klein CJ, Botuyan MV, Wu Y, Ward CJ, Nicholson GA, Hammans S, Hojo K, Yamanishi H, Karpf AR, Wallace DC, Simon M, Lander C, Boardman LA, et al. Mutations in DNMT1 cause hereditary sensory neuropathy with dementia and hearing loss. Nature genetics. 2011; 43:595-600.

11. Easwaran HP, Schermelleh L, Leonhardt H, Cardoso MC. Replication-independent chromatin loading of Dnmt1 during G2 and M phases. EMBO reports. 2004; 5:1181-1186.

12. Benetatos L, Vartholomatos G. On the potential role of DNMT1 in acute myeloid leukemia and myelodysplastic syndromes: not another mutated epigenetic driver. Annals of hematology. 2016; 95:1571-1582.

13. Chen CL, Yan X, Gao YN, Liao QP. Expression of DNA methyltransferase 1,3A and 3B mRNA in the epithelial ovarian carcinoma. Zhonghua fu chan ke za zhi. 2005; 40:770-774.

14. Zhu YM, Huang Q, Lin J, Hu Y, Chen J, Lai MD. Expression of human DNA methyltransferase 1 in colorectal cancer tissues and their corresponding distant normal tissues. International journal of colorectal disease. 2007; 22:661-666.

15. Peng DF, Kanai Y, Sawada M, Ushijima S, Hiraoka N, Kosuge T, Hirohashi S. Increased DNA methyltransferase 1 (DNMT1) protein expression in precancerous conditions and ductal carcinomas of the pancreas. Cancer science. 2005; 96:403-408.

16. Feng Y, Wang J, Wu Y, Feng X, Yu Y. Protein expressions of HDAC1 and DNMT1 in non-small-cell lung cancer and its clinical significance. Zhonghua yi xue za zhi. 2014; 94:596-598.

17. Ksiaa F, Ziadi S, Dhiab MB, Gacem RB, Trimeche M. Increased DNA methyltransferase 1 protein expression correlates significantly with intestinal histological type and gender in gastric carcinomas. Advances in medical sciences. $2015 ; 60: 50-57$.

18. Ksiaa F, Ziadi S, Gacem RB, Dhiab MB, Trimeche M. Correlation between DNA methyltransferases expression and Epstein-Barr virus, JC polyomavirus and Helicobacter pylori infections in gastric carcinomas. Neoplasma. 2014; 61:710-717. 
19. Cao XY, Ma HX, Shang YH, Jin MS, Kong F, Jia ZF, Cao DH, Wang YP, Suo J, Jiang J. DNA methyltransferase3a expression is an independent poor prognostic indicator in gastric cancer. World journal of gastroenterology. 2014; 20:8201-8208.

20. Ding WJ, Fang JY, Chen XY, Peng YS. The expression and clinical significance of DNA methyltransferase proteins in human gastric cancer. Digestive diseases and sciences. 2008; 53:2083-2089.

21. Etoh T, Kanai Y, Ushijima S, Nakagawa T, Nakanishi Y, Sasako M, Kitano S, Hirohashi S. Increased DNA methyltransferase 1 (DNMT1) protein expression correlates significantly with poorer tumor differentiation and frequent DNA hypermethylation of multiple $\mathrm{CpG}$ islands in gastric cancers. The American journal of pathology. 2004; 164:689-699.

22. Gao Y, Wang XC, Jiang X. Relationship between Promoter methylation of BNIP3 gene and DNMT1 expression in gastric cancer. Chinese Journal of Gastroenterology and Hepatology. 2016; 25:24-28.

23. Lu YO, Wang JP, Yuan N, Sun XC. Expression and Significance of PDCD4 and DNMT1 in Human Gastric Cancer. Chinese Journal of Clinical Laboratory Science. 2013; 31:702-704.

24. Liang YY, Lin JH. Preliminary research of PDCD4 and DNMT1 expression in human gastric cancer tissues. Experimental and Laboratory Medicine. 2015; 33:285-287.
25. Jiang XJ, Chu LL, Cui YX, Song MQ, Xie XJ, Tian YB. Relationship between promoter methylation of the Runx3 and Rassfla genes and Dnmt1 expression in gastric cancer. World Chinese Journal of Digestology. 2012; 20:3457-3463.

26. Sun N, Lu XF, Liu SN, Zheng XT, Li QQ. Expression and Significance of PDCD4 and DNMT1 in Gastric Carcinoma. Medicine and Philosophy (Clinical Decision Making Forum Edition). 2011; 32:54-56.

27. Liu B, Gu LP, Xing CP, Gao ZF, Sun QJ, Qian ZR, Dong L. Relationship between expression of Dnmt1 and Caveolin-1 in gastric cancer. Journal of the Fourth Military Medical University. 2009; 16:293-295.

28. Liu T, Cao MQ, Li XY, Yang WL. Expression of DNA methyltransferase 1 in human gastric cacner tissue and its significancer. Shanghai Medical Journal. 2007; 30:615-617.

29. Han J, He D, Feng ZY, Ding YG, Shao CK. Clinicopathologic features and protein expression study of Epstein-Barr virus-associated gastric carcinoma in Guangzhou. Chinese journal of pathology. 2010; 39:798 803.

30. Mutze K, Langer R, Schumacher F, Becker K, Ott K, Novotny A, Hapfelmeier A, Hofler H, Keller G. DNA methyltransferase 1 as a predictive biomarker and potential therapeutic target for chemotherapy in gastric cancer. European journal of cancer. 2011; 47:1817-1825. 